\title{
Treatment Efficacy of Semantic Feature Analyses for Persons with Aphasia: Evidence from Meta-Analyses
}

\author{
Se Jin Oh, Bora Eom, Chaewon Park, Jee Eun Sung \\ Department of Communication Disorders, Ewha Womans University, Seoul, Korea
}

Correspondence: Jee Eun Sung, PhD

Department of Communication Disorders, Ewha Womans University, 52 Ewhayeodae-gil, Seodaemun-gu, Seoul 03760, Korea

Tel: $+82-2-3277-2208$

Fax: +82-2-3277-2122

E-mail: jeesung@ewha.ac.kr

Received: April 5, 2016

Revised: May 15, 2016

Accepted: May 20, 2016

This work was supported by the BK21 Plus Project funded by the Ministry of Education of Korea.

\begin{abstract}
Objectives: Semantic feature analysis (SFA) is a treatment to improve word retrieval ability by strengthening impaired semantic networks. A large number of studies have reported the efficacy of SFA treatment on trained items, but its generalization effects on untrained items, discourse production or standardized language measures are controversial. Through the use of meta-analyses, the current study aimed to provide a systematic review of the treatment and generalization effects of SFA for individuals with aphasia. Methods: A systematic search based on 5 databases (DBPIA, RISS, EBSCOhost, ProQuest, PubMed) identified 11 studies which met the inclusion criteria. Effect sizes were calculated using Cohen's $d$ and robust improvement rate difference for naming and discourse production. We analyzed the standardized mean difference for standardized language measures. Results: Results demonstrated medium to large effect sizes for trained items, but small effect sizes for untrained items. For discourse production, effect sizes varied from small to large depending on the types of outcome measures. SFA treatment approach contributed to improving overall language ability, but its generalization effects onto naming domains from standardized language tests seem to be limited. Conclusion: SFA treatment was effective for improving naming ability on trained items and increasing overall language ability. However, its generalization effects were relatively limited for untrained items, discourse production and standardized naming tests. These results suggest that researchers and clinicians should consider several factors which may affect the treatment efficacy of a SFA approach for individuals with aphasia.
\end{abstract}

Keywords: Aphasia, Naming, Semantic feature analysis, Meta-analysis
실어증은 언어습득 이후 대뇌 언어 반구의 손상으로 인해 발생 하는 언어 장애이다(McNeil \& Pratt, 2001). 실어증의 여러 증상들 중에서 이름대기 장애는 중증도 및 실어증 유형과 상관없이 실어 증 환자들에게서 폭넓게, 지속적으로 나타나는 것으로 보고되고 있으며, 의사소통에 미치는 영향이 크다는 점에서 실어증 치료 분 야에서 중요한 이슈가 되어 왔다(Boyle, 2004; Coelho, McHugh, \& Boyle, 2000; Goodglass \& Baker, 1976; Goodglass \& Wingfield, 1997). 이름대기 장애는 의미 및 음운 등 다양한 네트워크의 손상 으로 인해 나타날 수 있으며(Butterworth, 1989; Caramazza, 1997; Dell, Schwartz, Martin, Saffran, \& Gagnon, 1997; Foygel \& Dell, 2000; Goldrick \& Rapp, 2007; Levelt, Roelofs, \& Meyer, 1999; Starreveld \& La Heij, 1996), 이에 대한 치료는 크게 의미 네트워크를 활
성화하는 방법과 음운치료에 집중하는 치료 방법으로 나뉘어져 시 행되어 왔다. 본 연구에서는 이 중에서도 의미 네트워크 활성화에 기반을 둔 치료 방법을 중점적으로 살펴보고자 한다.

이름대기 장애의 치료를 위해 의미 네트워크의 활성화에 중점을 두는 치료 접근법은 의미 네트워크가 개념을 구성하는 자질들 (conceptual features)로 이루어져 있으며, 단어를 인출하기 위해서 는 관련된 의미자질들이 활성화되어야 하고(Dell et al., 1997), 이러 한 의미자질들을 충분히 활성화시키지 못하거나 인출하는데 어려 움이 있을 때 이름대기 장애가 발생한다고 본다(Chapey, 2008; Wilshire \& Coslett, 2000). 이러한 점에서 이름대기 장애에 대한 치 료는 손상된 의미 네트워크를 강화시켜, 단어 인출 능력을 향상시 키고 이와 더불어 단어 인출의 어려움을 대체할 수 있는 의미적 처 
리 능력 또한 향상될 수 있도록 하는데 중점을 둔다(Raymer \& Rothi, 2001).

의미자질(semantic feature analysis, SFA) 중재는 의미적 접근의 대표적인 치료법 중의 하나로 목표 단어와 관련된 의미자질들을 산출하도록 함으로써 목표 단어 주변에 있는 의미 네트워크를 활 성화시켜 손상된 의미 체계의 회복을 유도하고, 목표 단어 인출의 가능성을 증가시키는데 목표를 둔다(Boyle \& Coelho, 1995; Coelho et al., 2000; Maddy, Capilouto, \& McComas, 2014). SFA 중재를 위해 임상가는 대상자에게 목표 단어를 나타내는 그림을 제시하며 이름대기를 요청한 후, 목표 단어와 의미적으로 연관되어 있는 의 미자질들을 산출하도록 유도한다. 이때 사용되는 의미자질들은 목 표 단어가 속한 상위 범주나 용도, 행위, 물리적 속성 등으로 목표 단어가 동일한 의미 범주에 속한 다른 항목들과 구별되게 하는 중 요한 자질들로 구성된다. 이처럼 목표 단어와 관련된 의미자질을 반복적으로 산출하도록 하는 것은 목표 단어로의 접근성을 향상 시키기 위해서일 뿐만 아니라 단어 인출이 어려운 상황에서 관련 된 의미자질로 접근하게 하는 전략의 사용을 자동화시키기 위함이 다(Boyle, 2004). 관련된 의미자질들을 산출한 후에는 다시 목표 단 어에 대한 이름대기를 요청하여 산출되는 단어의 정확도로 중재 효과를 평가한다. SFA 중재는 원래 외상성 뇌손상 환자들의 인지 및 의사소통 손상을 치료하기 위해 제안되었으며(Haarbauer-Krupa, Moser, Smith, Sullivan, \& Szekeres, 1985), Massaro와 Tompkins (1994)에서 의미자질 훈련에 대한 치료 효과가 보고되었다. 이 후, SFA 중재는 실어증 환자들의 이름대기 치료에도 도입되어 이름 대기 능력 향상에 효과가 있음이 보고되었다(Boyle, 2004; Boyle \& Coelho, 1995; Coelho et al., 2000; Conley \& Coelho, 2003; DeLong, Nessler, Wright, \& Wambaugh, 2015; Hashimoto \& Frome, 2011; Peach \& Reuter, 2010; Rider, Wright, Marshall, \& Page, 2008; Wambaugh, Mauszycki, Cameron, Wright, \& Nessler, 2013; Wambaugh, Mauszycki, \& Wright, 2014).

SFA 중재의 효과를 입증하려고 했던 연구자들은 중재 명사에 대한 치료 효과뿐만 아니라 일반화 효과에도 많은 관심을 보여왔 다. 이는 SFA 중재가 의미 네트워크를 강화시키는 것에 목표를 둔 다는 점에서 이미 여러 연구에 의해 검증된 중재 항목에 대한 이름 대기 능력뿐만 아니라 비중재 항목에 대한 이름대기 능력이나 담 화(discourse) 수준에서의 어휘-의미 정보처리 능력을 향상시키는 데에도 기여할 수 있다고 판단했기 때문이다(DeLong et al., 2015). 또한 SFA 접근법이 의사소통능력 전반의 향상에 목표를 두고 개발 되었고(Haarbauer-Krupa et al., 1985), 실어증 중재의 궁극적인 목 표가 자연스러운 맥락으로의 일반화(Thompson, 1989)라는 점을
고려해볼 때, SFA 중재로 인한 일반화 효과는 분석될 가치가 있다 고 볼 수 있다. SFA 중재의 일반화 효과를 알아보기 위해 연구자들 은 비중재 항목에 대한 이름대기 능력이나 담화 수준에서의 어휘의미 정보 산출능력, 또는 전반적인 언어능력에 미치는 영향에 관 심을 기울여 왔다. 그러나 이러한 일반화 효과에 대해서는 상이한 연구 결과들이 보고되어 왔다(Kiran, 2008; Raymer \& Rothi, 2001).

Boyle과 Coelho (1995)는 경도의 비유창 실어증 환자에게 SFA 중재를 실시하여 중재 및 비중재 이름대기 과제에서 나타난 효과 를 분석하였고, 그 결과로 중재 항목과 비중재 항목 모두에서 이름 대기 능력이 향상되었음을 밝히며 중재하지 않은 항목에 대한 일 반화 효과를 보고하였다. 이와 유사하게 Coelho 등(2000), Conley 와 Coelho (2003), Boyle (2004) 연구들에서도 SFA 중재를 실시한 후, 중재하지 않은 항목에 대해서 이름대기 수행력이 증가하는 현 상을 보고하였다. 그러나 Rider 등(2008)은 3명의 실어증 환자들을 대상으로 대상자들에게 익숙한 상황으로부터 중재 항목들을 설정 하여 SFA 중재를 실시한 후 중재 및 비중재 명사들에 대한 중재 효 과를 알아보았다. 그 결과, 중재 명사의 경우 이름대기 수행력이 증 가하는 현상을 보였으나, 비중재 명사에 대해서는 3 명의 대상자 모 두에게서 효과가 전혀 나타나지 않았거나 미미한 효과만 보이는 것 으로 나타났다. Wambaugh와 Ferguson (2007) 그리고 Kristensson, Behrns와 Saldert (2015) 연구에서도 비중재 항목에 대한 일반 화 효과는 제한적이었음을 보고하였다.

담화 수준에서의 일반화 효과는 일상 생활에서 언어 사용의 향 상을 더 직접적으로 예측할 수 있다는 점에서 SFA 치료의 효과를 평가하는데 중요하게 다루어져야 할 요소라 할 수 있다(Peach \& Reuter, 2010). 담화 수준에서의 의미 정보 산출에 미치는 영향을 다룬 연구들은 자발화 과제에서의 단어 및 유효 정보 단위(correct information unit, $\mathrm{CIU}$ ) 산출 능력에 중점을 두었으며 측정 항목의 종류에 따라서 상이한 결과들을 제시해왔다. Boyle (2004)은 명칭 실어증 환자와 베르니케 실어증 환자에게 SFA 중재를 실시한 후, 자발화 과제(Brookshire \& Nicholas, 1994)에서의 일반화 효과를 분석하였다. 분석 결과, 대상자에 따라 지연되어 산출되는 단어의 수가 감소하여 분당 산출되는 $\mathrm{CIU}$ 수가 증가하거나 총 $\mathrm{CIU}$ 수가 증가하는 효과가 나타났으나, 그 외의 항목에 대해서는 변화가 없 어 담화 수준에서의 SFA 중재로 인한 일반화 효과는 제한이 있음 을 보고하였다. Antonucci (2009) 및 Falconer와 Antonucci (2012) 연구에서는 SFA 그룹 중재를 실시하여 자발화 과제(Nicholas \& Brookshire, 1993)에서의 단어 및 CIU 산출의 정확도와 효율성을 평가하였다. 자발화 과제에서의 단어 산출 수, $\mathrm{CIU}$ 총 수, 분당 산 출된 $\mathrm{CIU}$ 수, 전체 정보당 $\mathrm{CIU}$ 비율, 명사 및 동사 산출 비율을 분 
석한 결과, 대상자에 따라서 수행의 향상을 보인 항목은 달랐으며, 몇몇 항목에 대해서만 향상이 나타났다. 이러한 결과를 통해 연구 자들은 SFA 중재가 개별 대상자에게 미치는 효과의 정도는 다르지 만 목표 어휘의 의미적 지식에 접근하는 정확도나 효율성에 기여하 는 바가 있음을 제안하였다. Rider 등(2008) 연구에서는 자발화 과 제에서 산출된 중재 단어의 수가 중재 전에 비해 증가하였으나 이 러한 현상이 이야기 다시말하기나 절차 묘사하기와 같은 과제 수행 에서는 나타나지 않았다고 주장하며 담화 수준으로의 일반화 효 과는 제한이 있음을 보고하였다.

SFA 중재가 표준화 검사의 수행에 미친 일반화 효과에 대해서도 문헌들은 다양한 결과를 보고하였다. 표준화 검사 점수 상에 향상 이 있었다고 보고한 문헌들이 많았으나 실시된 검사의 종류에 따 라 상이한 결과들이 보고되었다. Rider 등(2008)은 SFA 중재 후 웨 스턴 실어증 검사(Western Aphasia Battery, WAB; Kertesz, 1982), 보스톤 이름대기 검사(Boston Naming Test, BNT; Kaplan, Gooodglass, \& Weinstaub, 2001) 등의 표준화 검사의 수행력 변화를 살펴 보았는데, 전반적인 점수 상의 변화는 크지 않았으나 일부 대상자 들의 $\mathrm{WAB}$ 실어증 지수(Aphasia Quotient, $\mathrm{AQ}$ )와 $\mathrm{BNT}$ 점수에서 약간의 향상이 있었음을 제안하였다. Antonucci (2009)에서는 대 상자 모두에게서 $\mathrm{WAB} \mathrm{AQ}$ 점수가 소폭 증가하는 경향을 보였고, $\mathrm{BNT}$ 점수의 경우에는 점수 상의 변화는 크게 없었으나 의미적 접 근의 향상을 보여주는 오류 패턴 상의 질적인 변화를 보고하기도 하였다. 한편, Kiran (2008) 연구에서는 중재 항목의 수행력 변화와 비중재 항목 및 WAB AQ, BNT, Psycholinguistic Assessment of Language Processing in Aphasia (PALPA; Kay, Lesser, \& Coltheart, 1992), Pyramids and Palm Tree (PAPT; Howard \& Patterson, 1992) 점수 간의 상관관계를 분석하였다. 분석 결과, 한 명의 대상자에게 서 중재 항목의 이름대기 점수와 PALPA 및 $\mathrm{PAPT}$ 검사 점수 간에 유의한 상관관계가 있었음을 제시하며, SFA 중재가 표준화 검사수 행력 향상에 기여할 수 있지만 그 효과가 제한적임을 보고하였다.

이처럼 SFA 중재 효과에 관한 연구들은 중재 항목에 대해서는 효과가 있다는 비교적 일관성 있는 결과를 보여주고 있으나, 중재 하지 않은 과제 및 표준화 검사의 수행에 미치는 효과에 있어서는 상이한 결과를 제시하고 있다. 따라서 SFA 중재가 실어증 환자들의 어휘-의미 정보처리 능력의 향상에 어느 정도 기여하는지에 대한 종합적 결론을 도출하기에는 제한이 있다. 또한 이러한 SFA 중재의 효과를 보고한 연구들은 대부분 단일대상연구 설계로 진행되었고 연구간 실험 조건이 다르다는 점에서 개별 연구 결과를 종합한 효 과 정도를 파악하기가 어려운 점이 있다. Maddy 등(2014)은 SFA 중 재에 대한 체계적 문헌연구를 실시하였는데, 신경학적 손상을 동
반한 환자들에게 SFA 중재를 적용한 11 편의 연구들을 분석한 결과, SFA 중재가 중재 받은 항목에 대한 대면 이름대기 능력을 향상시키 는데 효과적인 중재법임을 보고하였다. 그러나 Maddy 등(2014)의 연구에서는 실어증뿐만 아니라 외상성 뇌손상 대상자들을 포함하 였으며, 중재하지 않은 과제 및 표준화 검사에서의 일반화 효과는 분석하지 않았다. 또한 효과크기를 측정하는 방식에 있어서 기초 선 단계에서의 이상치의 영향을 많이 받고 효과크기가 과대평가될 우려가 있는 비중복 비율(percentage of non-overlapping data, PND; Scruggs, Mastropieri, \& Casto, 1987) 방식을 이용하여 분석 결과를 제시하였다(Parker, Vannest, \& Brown, 2009). 따라서 본 연 구에서는 지금까지 실어증 환자들을 대상으로 SFA 중재 효과를 보고한 문헌들을 종합하여 SFA 중재의 치료 효과를 살펴보고, 비 중재 명사 이름대기, 담화 과제 및 표준화 검사 수행에 미치는 일반 화 효과를 분석하고자 하였다. 또한 이상치의 영향을 최소화할 수 있는 효과크기 측정법인 개선율 차이(improvement rate difference, IRD; Parker, Vannest, \& Davis, 2011) 방식을 보다 개선한 형 태인 robust IRD 방식을 적용하여 메타분석을 실시하였다. 본 연구 의 연구 질문은 다음과 같다.

첫째, SFA 중재가 실어증 환자의 중재 명사 이름대기에 미치는 효과는 어떠한가?

둘째, SFA 중재가 실어증 환자의 비중재 명사 이름대기에 미치는 일반화 효과는 어떠한가?

셋째, SFA 중재가 실어증 환자의 담화 수준에서의 단어 및 의미 정보 산출에 미치는 일반화 효과는 어떠한가?

넷째, SFA 중재가 실어증 환자의 표준화 검사 수행에 미치는 일 반화 효과는 어떠한가?

\section{연구 방법}

\section{문헌검색 및 선정}

실어증 환자들을 대상으로 SFA 중재 효과를 살펴보기 위하여 2016년 3월에 국내외 데이터베이스를 통해 관련 연구들을 수집하 였다. 문헌 선정 기준은 실어증 환자를 대상으로 명사 자극을 사용 하여 SFA 중재를 실시한 단일대상연구만을 분석 문헌으로 선정하 되 분석대상자는 뇌졸중 발병 후 6개월 이상이 경과한 단일언어 사용자로 발병 전 손잡이가 오른손잡이며 좌반구 뇌졸중으로 인 한 실어증 환자만을 포함하였다. 문헌검색을 위해 국외(EBSCOhost, ProQuest, PubMed) 3 개, 국내(DBPIA, RISS) 2 개, 총 5 개의 데 이터베이스를 활용하였다. 문헌검색 조건은 학술지 문헌으로 제한 하였으며 검색어는 국외 데이터베이스는 'aphasia and semantic 
feature analysis or SFA treatment or SFA therapy or SFA intervention'으로 국내 데이터베이스는 'aphasia and semantic feature analysis'으로 입력하여 문헌을 수집하였다.

문헌검색을 통해 PubMed에서 183편, ProQuest에서 88편, EBSCOhost에서 72편, RISS에서 10편, DBPIA에서 0편, 총 353편의 연 구가 검색되었다. 이중 중복된 문헌 48 편을 제외한 나머지 연구 305 편의 제목과 초록을 통해 SFA 중재와 관련성이 없는 연구 274 편을 추가로 제외하였다. 또한 연구대상이 실어증 환자가 아닌 문 헌 2편과 단일대상연구 외 실험설계 방법을 사용한 문헌 2편을 분 석에서 제외하였으며 출판형태가 리뷰에 해당하는 2 편, 대상자가 단일언어 사용자가 아닌 2편을 제외하였다. 이후, 나머지 23 편의 연 구 전문 확인을 통해 SFA 중재 외 다른 중재가 복합적으로 실시된 연구 6편, 중재자극이 명사가 아닌 경우(예: 동사) 4편을 제외하였 다. 또한 SFA 중재 효과를 살펴보았지만 언어 평가를 실시하지 않 은 경우 또는 그 값을 제시하지 않은 연구 2 편이 제외되었다. 최종 적으로 실어증 환자를 대상으로 SFA 중재를 실시한 단일대상연구 중 중재에 따른 언어 효과를 살펴본 총 11 편의 문헌이 선정되었다. 문헌 선정 및 제외 기준은 Table 1 에 제시하였고 선정 과정과 흐름 도는 Figure 1과같다.

\section{연구의 질 평가}

선정된 문헌의 질적 평가를 위해 Tate 등(2008)의 단일대상연구 (single case experimental design, SCED) 평가척도를 사용하였다.
평가항목으로 연구대상자 병력, 목표 행동에 대한 정확한 정의, 효 과적인 실험디자인, 안정적인 기초선 및 중재 효과 측정, 자료기록

Table 1. Criteria for inclusion and exclusion

\begin{tabular}{|c|c|c|}
\hline & Inclusion criteria & Exclusion criteria \\
\hline Research design & Single-subject design & $\begin{array}{l}\text { Group design } \\
\text { Qualitative research } \\
\text { Pre-post case study }\end{array}$ \\
\hline Participant & Aphasia due to stroke & $\begin{array}{l}\text { Sensory deficits } \\
\text { Severe dysarthria or apraxia } \\
\text { History of neurological disease } \\
\text { TBI } \\
\text { Right-hemisphere stroke } \\
\text { Left-handed } \\
\text { Multilingual } \\
\text { Less than } 6 \text { months since } \\
\text { stroke onset }\end{array}$ \\
\hline $\begin{array}{l}\text { Intervention } \\
\text { approach }\end{array}$ & SFA, modified SFA & $\begin{array}{l}\text { SFA with other intervention } \\
\text { approaches }\end{array}$ \\
\hline Stimuli & Noun & Verb \\
\hline $\begin{array}{l}\text { Dependent } \\
\text { measures }\end{array}$ & $\begin{array}{l}\text { WAB AO } \\
\text { BNT } \\
\text { Confrontation naming } \\
\text { Percentage of nouns retrieved } \\
\text { Percentage of verbs retrieved } \\
\text { Number of CIUs (average) } \\
\text { CIUs per minute } \\
\text { Percentage of CIUs }\end{array}$ & Other measures \\
\hline
\end{tabular}

$\mathrm{TBI}=$ traumatic brain injury; $\mathrm{SFA}=$ semantic feature analysis; $\mathrm{WAB}=$ Western Aphasia Battery (Kertesz, 1982, 2006); A0=Aphasia Quotient; BNT = Boston Naming Test (Kaplan, Goodglass, \& Weintraub, 1983, 2001); CIUs= correct information units.

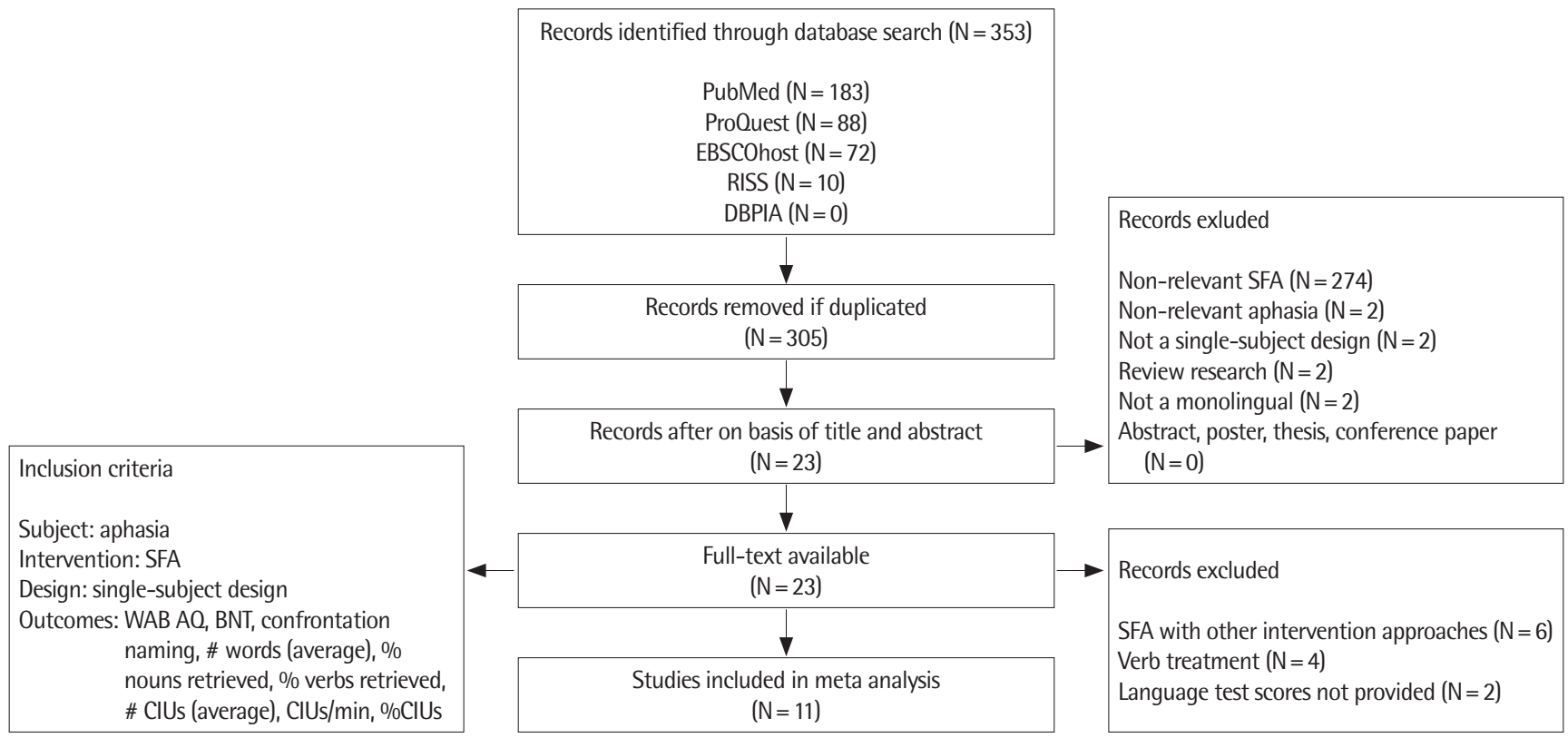

Figure 1. Flowchart of studies included from database search. $S F A=$ semantic feature analysis; WAB=Western Aphasia Battery (Kertesz, 1982, 2006); $A 0=A p h a s i a$ Quotient; BNT= Boston Naming Test (Kaplan, Goodglass, \& Weintraub, 1983, 2001); CIUs = correct information units. 
Table 2. Assessment of methodological quality of single-case experimental designs

\begin{tabular}{clc}
\hline No. & \multicolumn{1}{c}{ Title } & SCED quality score \\
\hline 1 & Antonucci (2009) & 8.5 \\
2 & Boyle (2004) & 10.5 \\
3 & Conley \& Coelho (2003) & 8 \\
4 & DeLong et al. (2015) & 10 \\
5 & Falconer \& Antonucci (2012) & 8 \\
6 & Hashimoto \& Frome (2011) & 9.5 \\
7 & Kiran (2008) & 10.5 \\
8 & Kiran \& Thompson (2003) & 10.5 \\
9 & Kristensson et al. (2015) & 10.5 \\
10 & Rider et al. (2008) & 10 \\
11 & Wambaugh et al. (2013) & 10.5 \\
\hline
\end{tabular}

SCED = single-case experimental design .

의 정확성, 평가자 간 신뢰도 실시, 독립된 평가자의 유무, 통계분석 실시, 반복측정 가능여부, 일반화 효과 등의 11 개의 항목을 분석하 였다. 각 항목에 대해 0 점(부적절)에서 1 점(적절)으로 판별을 하고 각 연구별로 총점을 산출하였다. 분석 결과 분석된 연구들의 점수 범위는 8점에서 10.5점, 평균 점수는 9.7점으로 나타나, 종합적인 분 석 논문의 대상으로 적합한 것으로 평가되었다(Lock \& Amstrong, 1997). 각 문헌별 결과는 Table 2에 제시하였다.

\section{코딩 및 자료 분석}

본 연구에서는 선정된 연구 11편의 SFA 중재 효과를 살펴보기 위해 각 문헌에서 제시한 중재 및 비중재 명사 이름대기 결과와 담 화 수준에서 자발화 산출 결과 값(평균 단어 산출 수, 명사 및 동사 산출 비율, 평균 $\mathrm{CIU}$ 수, 분당 $\mathrm{CIU}$ 수, $\mathrm{CIU}$ 비율)을 종속변인으로 살펴보았다. 각 과제에 따른 대상자별 효과크기는 선정된 연구에서 Cohen's $d$ (Beeson \& Robey, 2006)값이 제시된 경우 그 값을 사용 하였고, 그 값이 제시되지 않았거나 계산할 수 없는 경우 robust $\mathrm{IRD}$ 를 계산하여 분석하였다. Cohen's $d$ 는 중재 단계의 평균값에 서 기초선 단계의 평균값을 뺀 값을 기초선 평균표준편차 $\mathrm{SD}$ 로 나 눈 값을 말한다. Robust IRD는 더욱 신뢰성 있는 결과를 얻기 위하 여 IRD를 보완하여 변형한 것으로 기초선과 중재 단계의 중복된 자료점들을 각 단계에 동일하게 나눠준 후, 중재 단계에서 기초선 단계보다 향상된 정도로 계산된 값을 말한다(Parker et al., 2011). 효과크기의 해석을 위해 Cohen's $d$ 의 경우 Beeson과 Robey (2006) 의 기준을 적용하여 치료 효과크기를 높은(large, $d \geq 10.1$ ), 중간 (medium, $7.0 \leq d \leq 10.0$ ), 낮은(small, $4.0 \leq d \leq 6.9$ )으로 구분하였 다. Robust IRD의 경우 Parker 등(2009)의 기준을 적용하여 점수가 .5 미만은 효과크기가 의심스럽거나 낮은 효과로, .5-.7 미만은 중간
효과크기로, .7 이상은 높은 효과크기로 구분하였다.

SFA 중재가 표준화 언어검사 수행력에 미치는 효과를 분석하기 위하여 선정된 문헌에서 실어증 환자의 전반적인 언어능력을 측정 한 $\mathrm{WAB}$ 의 $\mathrm{AQ}$ 와 명사 대면이름대기 능력을 측정한 $\mathrm{BNT}$ 의 중재 전·후 점수를 종속변인으로 사용하였다. 효과크기는 통계프로그램 인 CMA2 (Comprehensive Meta-Analysis ver. 2)를 사용하여 분석 하였다. 각 문헌의 연구자, 출판년도, 독립변인(SFA 중재) 및 종속변 인(WAB AQ, BNT 점수)의 통계적 수치(중재 전·후 평균, 표준편차, 표본크기, $r$ 값)를 입력하여 분석하였다. 각 과제에 따른 연구결과별 효과크기는 표준화된 평균차이(standardized mean difference, SMD)를 산출하였으며, 95\% 신뢰수준(confidence intervals)을 계 산하였다. 선정된 문헌의 연구 방법, 표본 등이 다양하다는 점을 인 정하여 무선효과모형(random-effects model)을 적용하여 결과를 산출하였다(Borenstein, Hedges, Higgins, \& Rothstein, 2009).

\section{신뢰도 평가}

중재 및 중재 일반화 효과크기를 robust IRD로 계산한 경우 제 1 연구자와 제 2 연구자 간의 평가자 간 신뢰도를 산출하였다. 전체 문 헌 대상자의 robust IRD를 2 명의 저자가 개별적으로 계산하였을 때 일치율은 $100 \%$ 였다. CMA2를 사용하여 효과크기를 산출한 전 반적인 언어검사의 경우 총 3 명의 저자가 전체 11 편의 연구를 개별 적으로 코딩 후 효과크기를 산출한 결과 일치율은 $100 \%$ 였다.

\section{연구 결과}

\section{선정된 연구의 특성}

본 연구에서 분석된 논문은 총 11편이었으며, 각 연구별 대상자 의 수는 1 명에서 9 명으로 총 36 명의 실어증 환자들의 중재 결과가 본 연구에서 분석되었다. 대상자의 연령은 30 세에서 80 세까지 다 양했으며, 실어증 발병 기간 또한 7 개월부터 384 개월까지 다양한 분포를 보였다. 대상자들의 실어증 유형은 명칭실어증, 브로카, 베 르니케, 전도실어증, 연결피질운동실어증 등으로 다양한 유형의 대 상자들이 참여하였다. SFA 중재를 실시한 기간은 평균 12 주(범위: 5-28주), 중재 회기 수는 평균 30회(범위: 9-82회), 한 회기의 시간은 평균 75분(범위: $45-120$ 분)이었다.

SFA 중재의 효과를 측정하기 위하여 본 연구 주제와 관련하여 사용된 과제는 다음과 같다. 중재 및 비중재 항목에 대한 효과 분석 을 위해서 대면이름대기 과제가 사용되었으며, 종속 변수는 반응 의 정확도였다. 담화 수준에서의 일반화 효과 측정을 위해서는 그 림자극을 보고 설명하는 과제(Brookshire \& Nicholas, 1994; Da- 
Table 3. The effects of SFA on confrontation naming for treated and untreated items

\begin{tabular}{|c|c|c|c|c|c|c|c|c|}
\hline \multirow{2}{*}{ Study } & \multirow{2}{*}{ Participant } & \multirow{2}{*}{ Aphasia type } & \multicolumn{3}{|c|}{ Effect size for treated items } & \multicolumn{3}{|c|}{ Effect size for untreated items } \\
\hline & & & Cohen's $d^{\mathrm{a}}$ & Robust IRD ${ }^{b}$ & Magnitude of effect & Cohen's $d^{a}$ & Robust IRD & Magnitude of effect \\
\hline \multirow[t]{2}{*}{ Boyle (2004) } & p1 & Anomic & & 1 & Large & & 1 & Large \\
\hline & p2 & Wernicke & & .9 & Large & & 1 & Large \\
\hline Conley \& Coelho (2003) & p1 & Broca & & .8 & Large & & .79 & Large \\
\hline \multirow[t]{4}{*}{ DeLong et al. (2015) } & p1 & Conduction & 4.1 & & Small & 6.28 & & Small \\
\hline & p2 & Wernicke & 13.88 & & Large & 5.61 & & Small \\
\hline & p3 & Broca & 10.74 & & Large & 3.38 & & Marginally small \\
\hline & p4 & Anomic & 21.97 & & Large & 4.44 & & Small \\
\hline Hashimoto \& Frome (2011) & p1 & Broca & & .94 & Large & & .12 & Small \\
\hline \multirow[t]{5}{*}{ Kiran (2008) } & p1 & Conduction & & 1 & Large & & .86 & Large \\
\hline & p2 & Conduction & & 1 & Large & & .54 & Moderate \\
\hline & p3 & Conduction & & .6 & Moderate & & .29 & Small \\
\hline & p4 & Broca & & .67 & Moderate & & .5 & Moderate \\
\hline & p5 & Broca & & .43 & Small & & -.14 & - \\
\hline \multirow{4}{*}{ Kiran \& Thompson (2003) } & p1 & Fluent & & .78 & Large & & .65 & Moderate \\
\hline & p2 & Fluent & & 1 & Large & & .96 & Large \\
\hline & p3 & Fluent & & .84 & Large & & -.18 & - \\
\hline & p4 & Fluent & & .92 & Large & & .77 & Large \\
\hline \multirow[t]{3}{*}{ Kristensson et al. (2015) } & p1 & Wernicke & & .33 & Small & & -.5 & - \\
\hline & p2 & Mixed non-fluent & & .47 & Small & & -.17 & - \\
\hline & p3 & Broca & & .47 & Small & & .09 & Small \\
\hline \multirow[t]{3}{*}{ Rider et al. (2008) } & p1 & Transcortical motor & 3.86 & & Marginally small & -.88 & & - \\
\hline & p2 & Transcortical motor & 5.54 & & small & 1.21 & & Marginally small \\
\hline & p3 & Broca & 2.97 & & Marginally small & 1 & & Marginally small \\
\hline \multirow[t]{9}{*}{ Wambaugh et al. (2013) } & p1 & Broca & 5.32 & & Small & & & \\
\hline & p2 & Anomic & 7.95 & & Medium & & & \\
\hline & p3 & Broca & 9.28 & & Medium & & & \\
\hline & p4 & Broca & 7.48 & & Medium & & & \\
\hline & p5 & Broca & 1.26 & & Marginally small & & & \\
\hline & p6 & Broca & 5.01 & & Small & & & \\
\hline & p7 & Broca & 7.79 & & Medium & & & \\
\hline & p8 & Anomic & 7.32 & & Medium & & & \\
\hline & p9 & Wernicke & 9.62 & & Medium & & & \\
\hline
\end{tabular}

SFA = semantic feature analysis; $I R D=$ improvement rate difference.

aSmall effect, $4.0 \leq d \leq 6.9$; medium effect, $7.0 \leq d \leq 10.0$; large effect, $d \geq 10.1$.

${ }^{\mathrm{b}}$ Small effect, $\mathrm{IRD}<.5$; moderate effect, $.5 \leq \mathrm{IRD}<.7$; large effect, $\mathrm{IRD} \geq .7$.

vis, 2005; Nicholas \& Brookshire, 1993)가 사용되었으며, 종속 변 수는 평균 단어 산출수, 명사 및 동사산출 비율, 평균 $\mathrm{CIU}$ 수, 분당 산출된 CIU 수, $\mathrm{CIU}$ 비율이었다. 표준화 검사에서의 일반화 효과 측정을 위해서는 언어의 전반적인 능력을 측정하는 WAB (Kertesz, 1982, 2006)과 BNT (Kaplan, Goodglass, \& Weintraub, 1983, 2001) 가 사용되었으며 $\mathrm{WAB} \mathrm{AQ}$ 와 $\mathrm{BNT}$ 의 점수를 종속 변수로 사용하 였다. 개별연구에 대한 정보는 Appendix 1에 제시하였다.

\section{SFA 중재의 효과크기 분석}

SFA 중재가 중재 명사 이름대기에 미치는 영향

$\mathrm{SFA}$ 중재가 중재 명사의 이름대기에 미치는 평균 효과크기를 분 석한 결과, 개별 연구에서 제시된 Cohen's $d$ 점수를 기준으로 $d=7.76$
$(\mathrm{SD}=4.94, \mathrm{~N}=16)$ 의 중간 정도의 효과크기를 보이는 것으로 나타 났으며, 효과크기가 제시되지 않은 연구의 경우 robust IRD 점수를 분석한 결과 $.76(\mathrm{SD}=.23, \mathrm{~N}=16)$ 으로 높은 효과크기를 보이는 것 으로 나타났다(Table 3 ).

\section{SFA 중재가 비중재 명사 이름대기에 미치는 영향}

중재하지 않은 명사에 대한 일반화 효과를 분석한 결과, Cohen's $d$ 점수 기준 $d=3.01(\mathrm{SD}=2.65, \mathrm{~N}=7)$ 로 낮은 효과크기를 보이는 것으로 나타났고, robust IRD 점수 또한 .41 $(\mathrm{SD}=.49, \mathrm{~N}=16)$ 로 낮 은 효과크기를 보이는 것으로 나타났다. 이름대기 수행에 대한 각 연구의 대상자별 효과크기는 Table 3에 제시하였다. 
SFA 중재가 담화 수준에서의 단어 및 의미 정보 산출에 미치는 영향

SFA 중재가 담화 수준에서의 자발화 산출 과제에서 단어 산출 수에 미치는 영향을 분석한 결과, 총 단어 산출의 경우 robust IRD 점수가. $48(\mathrm{SD}=.42, \mathrm{~N}=4)$ 로 낮은 효과크기를 나타낸 반면, 산출 된 명사 및 동사의 비율은 모두 $.75\left(\mathrm{SD}_{\text {noun }}=.17, \mathrm{~N}=4 ; \mathrm{SD}_{\text {verb }}=.32\right.$,
$\mathrm{N}=4$ )로 높은 효과크기를 보이는 것으로 나타났다. CIU 관련 변인 의 경우, 자발화에서 산출된 의미 정보 중 평균 CIU 수에 대한 robust IRD 점수는 $.63(\mathrm{SD}=.43, \mathrm{~N}=6)$ 으로 중간 정도의 효과크기 를 보였으나, 분당 $\mathrm{CIU}$ 수 및 전체 의미 정보에 대한 CIU 비율의 점 수는 각각 $.48(\mathrm{SD}=.44, \mathrm{~N}=6)$ 과 $.44(\mathrm{SD}=.37, \mathrm{~N}=6)$ 로 낮은 효과

Table 4. The effectiveness of SFA on number of words, percentage of nouns retrieved, percentage of verbs retrieved

\begin{tabular}{|c|c|c|c|c|c|c|c|c|}
\hline \multirow[b]{2}{*}{ Study } & \multirow[b]{2}{*}{ Participant } & \multirow[b]{2}{*}{ Aphasia type } & \multicolumn{2}{|c|}{ Number of words (average) } & \multicolumn{2}{|c|}{ Percentage of nouns retrieved } & \multicolumn{2}{|c|}{ Percentage of verbs retrieved } \\
\hline & & & $\begin{array}{c}\text { Robust } \\
\text { IRD }^{\mathrm{a}}\end{array}$ & $\begin{array}{l}\text { Magnitude of } \\
\text { effect }\end{array}$ & $\begin{array}{l}\text { Robust } \\
\text { IRD }^{\mathrm{a}}\end{array}$ & $\begin{array}{l}\text { Magnitude of } \\
\text { effect }\end{array}$ & $\begin{array}{l}\text { Robust } \\
\text { IRD }^{\mathrm{a}}\end{array}$ & $\begin{array}{l}\text { Magnitude of } \\
\text { effect }\end{array}$ \\
\hline \multirow[t]{2}{*}{ Antonucci (2009) } & p1 & Conduction & 1 & Large & .67 & Moderate & .67 & Moderate \\
\hline & p2 & Anomic & 0 & - & .67 & Moderate & .33 & Small \\
\hline \multirow{2}{*}{$\begin{array}{l}\text { Falconer \& Antonucci } \\
\text { (2012) }\end{array}$} & p1 & Conduction & .33 & Small & .67 & Moderate & 1 & Large \\
\hline & p2 & Transcortical motor & .58 & Moderate & 1 & Large & 1 & Large \\
\hline
\end{tabular}

SFA= semantic feature analysis; $I R D=$ improvement rate difference.

a Small effect, IRD <.5; moderate effect, . $5 \leq \mathrm{IRD}<.7$; large effect, IRD $\geq .7$.

Table 5. The effectiveness of SFA on number of CIUs (average), CIUs per minute, percentage of CIUs

\begin{tabular}{|c|c|c|c|c|c|c|c|c|}
\hline \multirow[b]{2}{*}{ Study } & \multirow[b]{2}{*}{ Participant } & \multirow[b]{2}{*}{ Aphasia type } & \multicolumn{2}{|c|}{ Number of CIUs (average) } & \multicolumn{2}{|c|}{ CIUs per minute } & \multicolumn{2}{|c|}{ Percentage of CIUs } \\
\hline & & & $\begin{array}{l}\text { Robust } \\
\text { IRD }^{\mathrm{a}}\end{array}$ & $\begin{array}{l}\text { Magnitude of } \\
\text { effect }\end{array}$ & $\begin{array}{l}\text { Robust } \\
\text { IRD }^{\mathrm{a}}\end{array}$ & $\begin{array}{l}\text { Magnitude of } \\
\text { effect }\end{array}$ & $\begin{array}{c}\text { Robust } \\
\text { IRD }^{\mathrm{a}}\end{array}$ & $\begin{array}{l}\text { Magnitude of } \\
\text { effect }\end{array}$ \\
\hline Antonucci (2009) & $\begin{array}{l}\text { p1 } \\
\text { p2 }\end{array}$ & $\begin{array}{l}\text { Conduction } \\
\text { Anomic }\end{array}$ & $\begin{array}{l}1 \\
0\end{array}$ & $\begin{array}{c}\text { Large } \\
-\end{array}$ & $\begin{array}{l}0 \\
.17\end{array}$ & $\begin{array}{c}- \\
\text { Small }\end{array}$ & $\begin{array}{l}0 \\
1\end{array}$ & $\begin{array}{c}- \\
\text { Large }\end{array}$ \\
\hline Boyle (2004) & $\begin{array}{l}\text { p1 } \\
\text { p2 }\end{array}$ & $\begin{array}{l}\text { Anomic } \\
\text { Wernicke }\end{array}$ & $1^{.42}$ & $\begin{array}{l}\text { Small } \\
\text { Large }\end{array}$ & $\begin{array}{l}1 \\
.54\end{array}$ & $\begin{array}{c}\text { Large } \\
\text { Moderate }\end{array}$ & $\begin{array}{l}.13 \\
.54\end{array}$ & $\begin{array}{c}\text { Small } \\
\text { Moderate }\end{array}$ \\
\hline $\begin{array}{l}\text { Falconer \& Antonucci } \\
\text { (2012) }\end{array}$ & $\begin{array}{l}\text { p1 } \\
\text { p2 }\end{array}$ & $\begin{array}{l}\text { Conduction } \\
\text { Transcortical motor }\end{array}$ & $1^{.33}$ & $\begin{array}{l}\text { Small } \\
\text { Large }\end{array}$ & $\begin{array}{l}1 \\
.17\end{array}$ & $\begin{array}{l}\text { Large } \\
\text { Small }\end{array}$ & $\begin{array}{l}.67 \\
.33\end{array}$ & $\begin{array}{l}\text { Moderate } \\
\text { Small }\end{array}$ \\
\hline
\end{tabular}

SFA = semantic feature analysis; $\mathrm{CIUs}=$ correct information units; IRD = improvement rate difference.

${ }^{\text {aS }}$ mall effect, IRD <.5; moderate effect, . $5 \leq \mathrm{IRD}<.7$; large effect, $\mathrm{IRD} \geq .7$.

Study name

Antonucci(2009)
Antonucci(2009)
Falconer \& Antonucci(2012)
Falconer \& Antonucci(2012)
Kiran \& Thompson (2003)
Kiran \& Thompson (2003)
Kiran(2008)
Kiran(2008)
Rider(2008)
Rider(2008)

Outcome

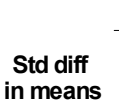

Statistics for each study

\begin{tabular}{lrrrrr}
\cline { 3 - 5 } & & \multicolumn{5}{c}{$\begin{array}{c}\text { Std diff } \\
\text { in means }\end{array}$} & $\begin{array}{c}\text { Lower } \\
\text { limit }\end{array}$ & $\begin{array}{c}\text { Upper } \\
\text { limit }\end{array}$ & Z-Value & $p$-Value \\
BNT & 0.025 & -0.037 & 0.087 & 0.794 & 0.427 \\
WAB_AQ & 0.112 & 0.049 & 0.174 & 3.519 & 0.000 \\
BNT & 0.032 & -0.019 & 0.082 & 1.224 & 0.221 \\
WAB_AQ & 0.025 & -0.025 & 0.076 & 0.985 & 0.325 \\
BNT & 1.421 & -0.614 & 3.456 & 1.368 & 0.171 \\
WAB_AQ & 0.655 & 0.213 & 1.098 & 2.902 & 0.004 \\
BNT & 0.960 & 0.346 & 1.575 & 3.065 & 0.002 \\
WAB_AQ & 0.677 & 0.382 & 0.972 & 4.503 & 0.000 \\
BNT & 0.330 & 0.010 & 0.651 & 2.021 & 0.043 \\
WAB_AQ & 0.071 & -0.089 & 0.232 & 0.873 & 0.383 \\
& 0.134 & 0.052 & 0.215 & 3.223 & 0.001
\end{tabular}

$\begin{array}{lllll}0.134 & 0.052 & 0.215 & 3.223 & 0.001\end{array}$

Figure 2. Forest plot of the effects of semantic feature analysis on standardized language tests for aphasia. 
Table 6. The effectiveness of SFA on standardized language tests for aphasia

\begin{tabular}{lccc}
\hline Type & SMD & $95 \%$ CI [LL, UL] & $p$-value \\
\hline Overall & .134 & $.052, .215$ & .001 \\
WAB A0 & .188 & $.052, .325$ & .007 \\
BNT & .098 & $-.021, .216$ & .107
\end{tabular}

SFA= semantic feature analysis; $\mathrm{SMD}=$ standardized mean difference; $\mathrm{Cl}=$ confidence interval; $\mathrm{LL}=$ lower limit; $\mathrm{UL}=$ upper limit; $\mathrm{WAB}=$ Western Aphasia Battery (Kertesz, 1982, 2006); $A 0=$ Aphasia Quotient; BNT =Boston Naming Test (Kaplan, Goodglass, \& Weintraub, 1983, 2001)

크기를 보이는 것으로 나타났다. 담화 수준에서의 단어 및 의미 정 보 산출과 관련된 각 연구의 대상자별 효과크기는 Tables 4 와 5에 제시하였다.

\section{SFA 중재가 표준화 검사 수행에 미치는 영향}

$\mathrm{SFA}$ 중재가 전반적인 언어능력의 변화에 미치는 영향을 알아보 기 위해 대상자들의 사전-사후 차이의 평균, 상관계수, 표본크기를 이용하여 표준화된 평균차이를 산출한 결과를 forest plot으로 제 시하였다(Figure 2). 분석 결과, 효과크기 $\mathrm{SMD}=.134, p<.01,95 \%$ 신뢰구간[.052, .215]으로 SFA 중재가 표준화 검사의 수행에 미치는 효과가 유의한 것으로 나타났다. 분석에 포함된 검사의 유형별로 분석한 결과, $\mathrm{WAB} \mathrm{AQ}$ 점수에 미치는 효과는 효과크기 $\mathrm{SMD}=.188$, $p<.01,95 \%$ 신뢰구간[.052, .325]으로 유의한 것으로 나타났으나, $\mathrm{BNT}$ 점수에 미치는 효과는 효과크기 $\mathrm{SMD}=.098, p>.05,95 \%$ 신 뢰구간[-.021, .216]으로 유의하지 않은 것으로 나타났다(Table 6).

\section{논의 및 결론}

본 연구는 메타분석 방법을 사용하여 실어증 환자들에게 실시 된 SFA 중재가 이름대기에 미치는 치료 효과를 살펴보고, 중재하 지 않은 과제 및 표준화 검사의 수행에 미치는 일반화 효과를 검증 하고자 하였다. 문헌 연구 포함 기준에 따라 11 개의 연구를 분석한 결과, 종속 변수에 따라 상이한 효과크기가 산출되었다. 중재 항목 에 대한 치료 효과는 중간-높은 수준의 효과크기를 보인 반면, 일 반화 효과에 대해서는 분석된 측정치에 따라 다양한 결과가 도출 되었다.

$\mathrm{SFA}$ 중재가 중재 항목의 이름대기 수행력에 미치는 효과가 높다 는 결과는 SFA 중재가 이름대기 향상에 효과가 있다는 기존의 연 구 결과 및 이에 대한 체계적 문헌 분석을 실시한 Maddy 등(2014) 의 연구와 일치한다. 이러한 결과는 목표 단어와 관련이 있는 의미 자질들을 반복해서 산출하도록 하여 목표 단어 주변의 의미 네트 워크를 활성화시키는 SFA 중재 방법이 이름대기 치료에 유용하다
는 것을 뒷받침한다(Boyle, 2004).

반면, 비중재 항목의 이름대기에 관해서는 일반화 효과가 낮은 것으로 나타났다. 이는 비중재 명사에 대한 일반화 효과를 보고한 연구(Boyle, 2004; Boyle \& Coelho, 1995; Coelho et al., 2000)와 일 반화 효과가 제한적이었음을 보고한 연구(Pring, Hamilton, Harwood, \& Macbride, 1993; Rider et al., 2008)가 혼재된 양상을 반영 한 결과로 보인다. 비중재 항목에 대한 일반화 효과가 일어나지 않 은 부분에 대해서는 다양한 요인들로 설명될 수 있다. DeLong 등 (2015)은 프로브(probe) 동안 비중재 명사에 노출된 횟수가 많을수 록, 목표 단어들이 속한 범주가 다양할수록 비중재 명사에 대한 일 반화가 더 촉진된다고 하였다. 또한 Kiran과 Thompson (2003)은 중재 항목과 비중재 항목의 의미적 연관성이 높을수록 일반화가 잘 일어난다고 주장하였고, Coelho 등(2000)은 대상자의 언어적인 특성이 일반화에 영향을 미칠 수 있음을 제안하였다. 본 연구에서 분석된 문헌들의 경우, 연구에 따라 비중재 명사에 노출된 횟수나 중재 범주가 다양하였고, 중재 항목과 비중재 항목의 의미적 연관 성 또한 연구들마다 상이하였다. 또한 대상자들의 실어증 유형이 다양했다는 점에서 이들의 언어적 특성 및 개인차(individual variability)가 일반화 효과에 영향을 주었을 것으로 생각된다. 이러한 여러 가지 변수들이 일반화 효과에 영향을 미쳐 비일관적인 효과 크기가 도출되었을 가능성이 있다.

담화 수준으로의 일반화 효과에 대해서는 분석된 항목별로 다양 하게 나타났으나, 효과크기가 크지 않은 경향을 보였다. 명사와 동사 의 산출 비율은 대체로 향상된 결과를 보였지만, 단어 산출 수나 유 효 정보 단위 관련 변수에 있어서는 효과크기가 비교적 작았다. 본 연구에서 분석된 문헌들 중 담화 맥락에서의 일반화 효과를 다룬 문헌은 단어 산출의 경우 2 편, $\mathrm{CIU}$ 산출의 경우에는 3 편에 불과하 여 본 연구의 결과만으로 이에 대한 일반화 효과를 단정짓기는 어렵 지만, 개별 단어 중재를 통해 담화 수준에서의 수행력 향상을 기대 하기 어렵다는 것은 여러 선행 연구들을 통해 제안된 바 있다(DeLong et al., 2005). Goodglass (1998)는 대면이름대기 또는 정의하기 과제가 자발화에서의 단어 인출을 촉진하기에 적합하지 않다는 제 안을 하였다. 또한 Ledoux, Camblin, Swaab 그리고 Gordon (2006) 에 따르면, 그림 설명하기와 같은 담화 수준 과제에서는 문맥을 통 해 유추 가능한 단서들을 활용할 수 있는 반면, 대면이름대기와 같 은 단어 수준의 과제에서는 문맥에서 오는 의미 점화(semantic priming in context)와 같은 관련 정보가 매우 제한적이다. 따라서, 담화수준으로의 일반화 효과를 기대하기 위해서는 의미 맥락이 있 는 과제를 활용한 중재가 필요하다고 주장하였다. 실제로 Mayer와 Murray (2003) 및 Peach와 Reuter (2010)는 담화수준에서 SFA 중재 
를 실시했을 때, 단어 수준에서의 중재에 비해 담화 수준에서의 단 어 인출 능력이 훨씬 더 향상되었다는 결과를 보고하였다.

표준화 검사에 대한 일반화 효과는 언어 전반적인 능력 부분이 나 실어증 지수에서 유의한 향상이 관찰되었으나, 이름대기 과제에 서는 유의한 효과가 나타나지 않았다. SFA 중재 후 표준화 검사에 서의 향상을 보고한 연구들은 이러한 일반화 효과가 SFA 중재 과정 에서 반복적으로 이루어지는 의미 정보의 처리 및 의미자질의 판단 을 통해 전반적인 의미 처리 능력이 향상된 것에 기인한다고 주장하 였다(Kiran, 2008; Kiran \& Thompson, 2003). 실제로 Kiran (2008) 은 표준화 검사 하위 영역들 중 향상을 보인 영역들은 의미자질이 나 항목 간의 관계를 판단하는 검사들이었음을 보고하면서, SFA 중재가 전반적인 의미처리 능력의 향상에 기여하는 유용한 중재법 임을 제안하였다. 중재를 통해 향상된 이름대기 능력이 표준화된 대면이름대기 검사의 항목으로 일반화되지 못한 결과와 관련하여 Kiran과 Thompson (2003)은 SFA 중재의 중재된 범주 외의 항목으 로의 일반화 효과는 제한이 있다고 주장하였다. SFA 중재의 특성 상 특정 범주의 단어에 집중하여 중재가 진행되기 때문에 대상자 들은 해당 범주의 단어에만 노출이 되어 다양한 범주가 제공되는 이름대기 과제에서는 일반화 효과를 기대하기 어렵다는 것이다.

본 연구는 SFA 중재의 치료 효과 및 일반화 효과를 보고한 연구 들을 체계적으로 분석하여 SFA 중재가 실어증 환자들을 대상으로 중재 명사의 이름대기 능력을 향상시키는데 높은 효과를 보이는 중 재법임을 보여주었다. 그러나 비중재 명사에 대한 일반화 효과는 낮은 것으로 나타났으며, 담화 수준 및 표준화 검사에 관해서는 항 목별로 효과크기가 상이하다는 통합된 결론을 도출하였다. 담화 수준에서 결과 분석에 포함된 연구가 2-3편으로 상대적으로 제한 적임을 고려할 때, 이에 대한 일반화된 결과 해석에는 제한점이 있 다. 또한 본 연구에서 종속 변수로 본 여러 영역들의 연구 수가 달라 영역별 효과크기에 대한 정확한 비교에는 한계가 있다. 본 연구에 포함된 대상자들은 3 명을 제외하고는 모국어가 영어인 실어증 환 자들이었기 때문에 언어적 다양성이 반영된 일반화된 결과를 도출 하지 못하였다. 본 연구의 결과는 영어라는 언어적 특수성이 반영 된 결과일 수 있다는 점에서 한국어 실어증 환자들에게 본 연구의 결과를 적용시키는 데에는 주의가 필요하다.

그럼에도 불구하고 본 연구는 실어증 치료에서 큰 비중을 차지하 고 있는 이름대기 중재법 중, SFA의 치료 효과크기에 대한 기초 자 료를 제시하였다는 점에서 근거기반 치료(evidence-based practice) 확립에 기여할 수 있을 것으로 기대된다. 또한 본 연구에서 제시된 SFA 중재의 제한된 일반화 효과에 대한 결과는 시간과 비용의 제 약이 따르는 치료 환경에서 중요하게 고려되어야 할 요소라고 판단
된다. 예컨대, 이름대기 능력의 향상을 넘어선 일반화 효과를 목표 로 SFA 중재를 적용하는 것에는 신중한 고려가 필요할 것이다. SFA 중재의 효과를 분석할 때, 본 연구에서 제시하였던 일반화에 미치 는 여러 요인들, 즉, 대상자의 특성, 중재 및 비중재 항목의 의미 범 주, 비중재 항목에 노출되는 횟수 등을 고려한 통합적인 판단도 필 요할 것으로 보인다. 또한 SFA 중재의 종합적인 일반화 효과에 대해 신뢰도 높은 결과를 얻기 위해서는 이러한 요인들이 치료 및 일반 화 효과에 미치는 구체적인 영향들에 대한 추가적인 연구가 진행되 어야할 것이다.

\section{REFERENCES}

Antonucci, S. M. (2009). Use of semantic feature analysis in group aphasia treatment. Aphasiology, 23, 854-866.

Beeson, P. M., \& Robey, R. R. (2006). Evaluating single-subject treatment research: lessons learned from the aphasia literature. Neuropsychology Review, 16, 161-169.

Borenstein, M., Hedges, L. V., Higgins, J., \& Rothstein, H. R. (2009). Introduction to meta-analysis. Chichester, UK: John Wiley \& Sons Ltd.

Boyle, M. (2004). Semantic feature analysis treatment for anomia in two fluent aphasia syndromes. American Journal of Speech-Language Pathology, 13, 236-249.

Boyle, M., \& Coelho, C. A. (1995). Application of semantic feature analysis as a treatment for aphasic dysnomia. American Journal of Speech-Language Pathology, 4, 94-98.

Brookshire, R. H., \& Nicholas, L. E. (1994). Test-retest stability of measures of connected speech in aphasia. Clinical Aphasiology, 22, 19-133.

Butterworth, B. (1989). Lexical access in speech production. In W. MarslenWilson (Ed.), Lexical representation and process (pp. 108-135). Cambridge, MA: MIT Press.

Caramazza, A. (1997). How many levels of processing are there in lexical access? Cognitive Neuropsychology, 14, 177-208.

Chapey, R. (2008). Language intervention strategies in aphasia and related neurogenic communication disorders (5th ed.). Baltimore, MD: Lippincott Williams \& Wilkins.

Coelho, C. A., McHugh, R. E., \& Boyle, M. (2000). Semantic feature analysis as a treatment for aphasic dysnomia: a replication. Aphasiology, 14, 133142.

Comprehensive Meta-Analysis (Version 2) [Computer software]. Englewood, NJ: Biostat. 
Conley, A., \& Coelho, C. (2003). Treatment of word retrieval impairment in chronic Broca’s aphasia. Aphasiology, 17, 203-211.

Davis, G. A. (2005). PACE revisited. Aphasiology, 19, 21-38.

Dell, G. S., Schwartz, M. F., Martin, N., Saffran, E. M., \& Gagnon, D. A. (1997). Lexical access in aphasic and nonaphasic speakers. Psychological Review, 104, 801-838.

DeLong, C., Nessler, C., Wright, S., \& Wambaugh, J. (2015). Semantic feature analysis: further examination of outcomes. American Journal of SpeechLanguage Pathology, 24, S864-S879.

Falconer, C., \& Antonucci, S. M. (2012). Use of semantic feature analysis in group discourse treatment for aphasia: extension and expansion. Aphasiology, 26, 64-82.

Foygel, D., \& Dell, G. S. (2000). Models of impaired lexical access in speech production. Journal of Memory and Language, 43, 182-216.

Goldrick, M., \& Rapp, B. (2007). Lexical and post-lexical phonological representations in spoken production. Cognition, 102, 219-260.

Goodglass, H. (1998). Stages of lexical retrieval. Aphasiology, 12, 287-298.

Goodglass, H., \& Baker, E. (1976). Semantic field, naming, and auditory comprehension in aphasia. Brain and Language, 3, 359-374.

Goodglass, H., \& Wingfield, A. (1997). Anomia: neuroanatomical and cognitive correlates. San Diego, CA: Academic Press.

Haarbauer-Krupa, J., Moser, L., Smith, G., Sullivan, D. M., \& Szekeres, S. F. (1985). Cognitive rehabilitation therapy: middle stages of recovery. In M. Ylvisaker (Ed.), Head injury rehabilitation: children and adolescents (pp. 287-310). San Diego, CA: College-Hill Press.

Hashimoto, N., \& Frome, A. (2011). The use of a modified semantic features analysis approach in aphasia. Journal of Communication Disorders, 44, 459469.

Howard, D., \& Patterson, K. E. (1992). The Pyramids and Palm Trees Test: a test of semantic access from words and pictures. Suffolk, UK: Thames Valley Test Company.

Kaplan, E., Goodglass, H., \& Weintraub, S. (1983). Boston Naming Test. Philadelphia, PA: Lea \& Febiger.

Kaplan, E., Goodglass, H., \& Weintraub, S. (2001). Boston Naming Test (2nd ed.). Philadelphia, PA: Lippincott Williams \& Wilkins.

Kay, J., Lesser, R., \& Coltheart, M. (1992). PALPA: psycholinguistic assessments of language processing in aphasia. East Sussex, UK: Psychology Press.

Kertesz, A. (1982). The Western Aphasia Battery. San Antonio, TX: Psychological Corporation.

Kertesz, A. (2006). The Western Aphasia Battery-revised. San Antonio, TX:
Psychological Corporation.

Kiran, S. (2008). Typicality of inanimate category exemplars in aphasia treatment: further evidence for semantic complexity. Journal of Speech, Language, and Hearing Research, 51, 1550-1568.

Kiran, S., \& Thompson, C. K. (2003). The role of semantic complexity in treatment of naming deficits: training semantic categories in fluent aphasia by controlling exemplar typicality. Journal of Speech, Language, and Hearing Research, 46, 608-622.

Kristensson, J., Behrns, I., \& Saldert, C. (2015). Effects on communication from intensive treatment with semantic feature analysis in aphasia.Aphasiology, 29, 466-487.

Ledoux, K., Camblin, C. C., Swaab, T. Y., \& Gordon, P. C. (2006). Reading words in discourse: the modulation of lexical priming effects by messagelevel context. Behavioral and Cognitive Neuroscience Reviews, 5, 107-127.

Levelt, W. J., Roelofs, A., \& Meyer, A. S. (1999). A theory of lexical access in speech production. Behavioral and Brain Sciences, 22, 1-38.

Lock, S., \& Armstrong, L. (1997). Cohesion analysis of the expository discourse of normal, fluent aphasic and demented adults: a role in differential diagnosis? Clinical Linguistics \& Phonetics, 11, 299-317.

Maddy, K. M., Capilouto, G. J., \& McComas, K. L. (2014). The effectiveness of semantic feature analysis: an evidence-based systematic review. Annals of Physical and Rehabilitation Medicine, 57, 254-267.

Massaro, M., \& Tompkins, C. A. (1994). Feature analysis for treatment of communication disorders in traumatically brain-injured patients: an efficacy study. Clinical Aphasiology, 22, 245-256.

Mayer, J., \& Murray, L. (2003). Functional measures of naming in aphasia: word retrieval in confrontation naming versus connected speech. Aphasiology, 17, 481-497.

McNeil, M. R., \& Pratt, S. R. (2001). Defining aphasia: some theoretical and clinical implications of operating from a formal definition. Aphasiology, 15, 901-911.

Nicholas, L. E., \& Brookshire, R. H. (1993). A system for quantifying the informativeness and efficiency of the connected speech of adults with aphasia. Journal of Speech, Language, and Hearing Research, 36, 338-350.

Parker, R. I., Vannest, K. J., \& Brown, L. (2009). The improvement rate difference for single-case research. Exceptional Children, 75, 135-150.

Parker, R. I., Vannest, K. J., \& Davis, J. L. (2011). Effect size in single-case research: a review of nine nonoverlap techniques. Behavior Modification, 35, 303-322.

Peach, R. K., \& Reuter, K. A. (2010). A discourse-based approach to semantic 
feature analysis for the treatment of aphasic word retrieval failures. Aphasiology, 24, 971-990.

Pring, T., Hamilton, A., Harwood, A., \& Macbride, L. (1993). Generalization of naming after picture/word matching tasks: only items appearing in therapy benefit. Aphasiology, 7, 383-394.

Raymer, A. M., \& Rothi, L. J. G. (2001). Cognitive approaches to impairments of word comprehension and production. In R. Chapey (Ed.), Language intervention strategies in aphasia and related neurogenic communication disorders (4th ed., pp. 524-550). Baltimore, MD: Lippincott Williams \& Wilkins.

Rider, J. D., Wright, H. H., Marshall, R. C., \& Page, J. L. (2008). Using semantic feature analysis to improve contextual discourse in adults with aphasia. American Journal of Speech-Language Pathology, 17, 161-172.

Scruggs, T. E., Mastropieri, M. A., \& Casto, G. (1987). The quantitative synthesis of single-subject research methodology and validation. Remedial and Special Education, 8, 24-33.

Starreveld, P. A., \& La Heij, W. (1996). Time-course analysis of semantic and orthographic context effects in picture naming. Journal of Experimental Psychology: Learning, Memory, and Cognition, 22, 896-918.

Tate, R. L., Mcdonald, S., Perdices, M., Togher, L., Schultz, R., \& Savage, S. (2008). Rating the methodological quality of single-subject designs and n-of-1 trials: introducing the Single-Case Experimental Design (SCED) Scale. Neuropsychological Rehabilitation, 18, 385-401.

Thompson, C. K. (1989). Generalization in the treatment of aphasia. In L. V. McReynolds \& J. E. Spradlin (Eds.), Generalization strategies in the treatment of communication disorders (pp. 82-115). St. Louis, MO: Mosby.

Wambaugh, J. L., \& Ferguson, M. (2007). Application of semantic feature analysis to retrieval of action names in aphasia. Journal of Rehabilitation Research and Development, 44, 381-394.

Wambaugh, J. L., Mauszycki, S., \& Wright, S. (2014). Semantic feature analysis: application to confrontation naming of actions in aphasia. Aphasiology, 28, 1-24.

Wambaugh, J. L., Mauszycki, S., Cameron, R., Wright, S., \& Nessler, C. (2013). Semantic feature analysis: incorporating typicality treatment and mediating strategy training to promote generalization. American Journal of SpeechLanguage Pathology, 22, S334-S369.

Wilshire, C. E., \& Coslett, H. B. (2000). Disorders of word retrieval in aphasia: theories and potential applications. In S. E. Nadeau, L. J. Gonzales-Rothi, \& B.Crosson (Eds.), Aphasia and language: theory to practice (pp. 82-107). New York: Guilford Press. 


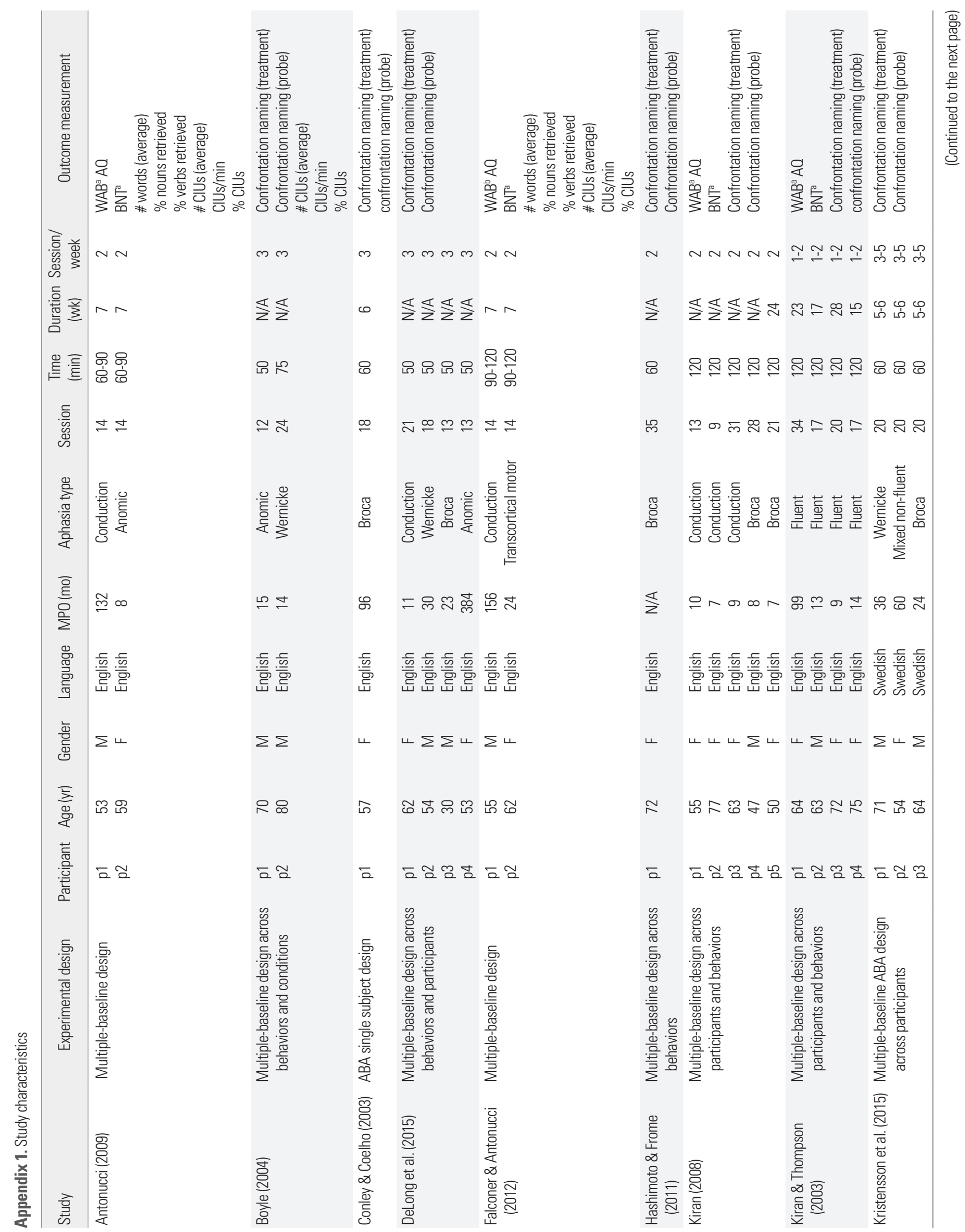




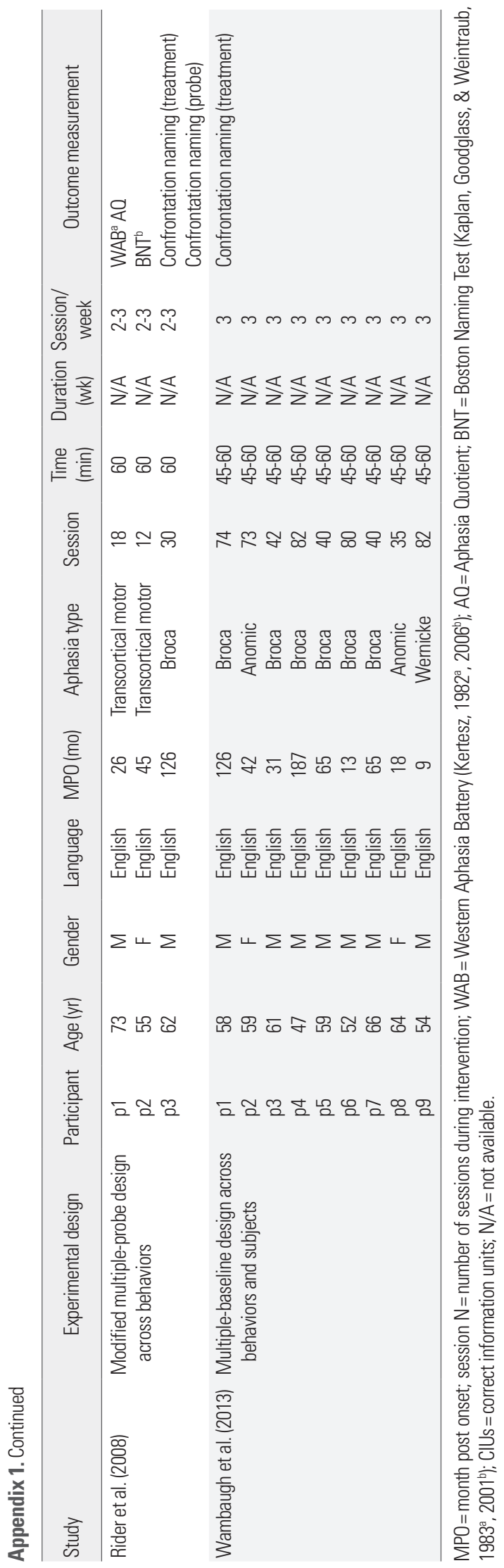




\section{국문초록}

\section{실어증 환자를 위한 의미자질 이름대기 중재 효과에 관한 메타 분석}

오세진 · 엄보라 · 박채원 · 성지은

이화여자대학교 언어병리학과

배경 및 목적: 의미자질(semantic feature analysis, SFA) 중재는 의미 네트워크를 강화시켜 단어 인출 능력을 향상시키는 실어증 이름대 기 중재법으로 중재 명사에 있어서는 비교적 일관적인 치료 효과가 보고되고 있으나, 일반화 효과에 대해서는 상이한 결과가 보고되고 있다. 따라서 본 연구는 SFA 중재를 실시한 문헌들을 체계적으로 분석하여 치료 및 일반화 효과에 대한 종합적인 결과를 도출하고자 하였다. 방법: 5 개의 국내외 데이터베이스에서 기준에 적합한 11 개의 논문을 선정하여 중재 및 비중재 명사와 자발화에 대해서는 Cohen's $d$ 와 개선율 차이(improvement rate difference)를, 표준화 언어검사에 대해서는 표준화된 평균차이(standardized mean difference)를 사용하여 효과크기를 산출하였다. 결과: 중재 명사에서는 중간에서 높은 수준의 효과크기가 산출되어 SFA 중재가 이름대기 치료에 효과가 있는 것으로 나타났으나 비중재 명사에서는 낮은 효과크기가, 자발화에서는 측정치에 따라 다양한 효과크기가 산출되 어 일반화 효과는 제한적인 것으로 나타났다. 또한 표준화 검사에서는 전반적인 언어능력 향상에는 유의한 효과가 있었으나 이름대기 검사에 미치는 효과는 유의하지 않았다. 논의 및 결론: 본 연구는 SFA 중재가 이름대기 및 전반적인 언어능력 향상에 긍정적인 영향을 미치는 치료법이나 비중재 자극으로의 일반화는 제한적임을 검증하였다. 이는 치료 및 일반화에 개입될 수 있는 다양한 변인들에 대한 추가적 연구와 더불어 임상적 차원에서의 고려가 필요함을 시사한다.

핵심어: 실어증, 이름대기, 의미자질 중재, 메타분석

본 연구는 BK21 Plus 사업의 지원을 받았음. 